\title{
CALCUL DE LA RÉPARTITION DES SURFACES D'ÉTANG EN CYPRINICULTURE
}

\author{
J. WIENIAWSKI*, B. LANOISELEE **
}

\footnotetext{
* Institut des Pêches Continentales, Division Pisciculture, Zabieniec, 05-500 PIASECZNO, Pologne.

* CEMAGREF, Groupement de Montpellier, Section Aménagements Littoraux et Aquaculture, B.P. 5095, 34033 MONTPELLIER CEDEX, France.
}

\section{RÉSUME}

II n'existe, à l'heure actuelle, aucune méthode précise et rapide pour calculer à l'avance la répartition rationnelle des surfaces d'étang destinées aux différentes phases d'élevage de la carpe. Cet article présente un mode de calcul satisfaisant au point de vue de la pratique et quatre exemples sont envisagés représentant différents programmes d'aménagement d'une pisciculture.

\section{COMPUTATION OF SURFACE-AREA ALLOCATION IN CARP POND FARMING}

\section{SUMMARY}

Nowadays, there is no rapid and precise method for calculating a rational allocation of pond area for different stages of carp culture. This report gives a method technically valuable and four different strategies of production are described in example.

\section{1 - INTRODUCTION}

La production de carpe en pisciculture d'étang se déroule en plusieurs étapes, chacune réalisée dans différentes catégories d'étangs selon la technologie utilisée. Pour assurer la continuité et la stabilité d'un programme de production au sein d'une exploitation, il est nécessaire d'adapter la production d'une phase d'élevage aux nécessités de la mise en charge de la phase suivante; ces lots de poissons de peuplement doivent correspondre à des critères qualitatifs et quantitatifs bien définis.

Ceci nécessite donc une répartition rationnelle du potentiel de production des surfaces disponibles. Le problème se pose de façon cruciale pour un projet de création de pisciculture, mais aussi lors de la réorganisation d'une installation existante (WIENIAWSKI, 1980). Dans la littérature, de telles méthodes de calculs n'existent pas ou restent très imprécises (WALTER, 1912, 1934 ; SPICZAKOW, 1932 ; SCHÄPERCLAUS, 1934), ou encore demandent des calculs prolongés (WIENIAWSKI, 1963).

Ce document a pour but de proposer une méthode simple et rapide pour ce calcul prévisionnel de répartition des surfaces destinées aux différentes phases d'élevage en carpiliculture: alevinage, $1^{\mathrm{er}}, 2^{\mathrm{e}}$ été ou grossissement, $3^{\mathrm{e}}$ été ou engraissement et hivernage.

\section{2 - PRINCIPE DE LA MÉthode}

La quantité de poissons obtenue à la fin d'un cycle de production composé de plusieurs phases dépend de la croissance individuelle et du taux de survie des sujets au cours de chacune des phases d'élevage ainsi que des rendements moyens observés dans les étangs où se déroulent ces phases (par rendement, il faut entendre biomasse totale récoltée).

Pour assurer la continuité des approvisionnements en sujets de peuplement de taille adaptée, il faut donc prévoir pour chaque étape une mise en charge tenant compte de ces trois paramètres inhérents au système de production (cycle de 2 ou 3 étés) et à la technicité mise en œuvre (fertilisation, alimentation, alevinage en 1 ou 2 phases, ...).

Ces trois paramètres sont intégrés dans une série de calculs et de simples opérations arithmétiques permettent d'obtenir les résultats désirés selon les options retenues sur l'exploitation. 


\section{3 - DÉROULEMENT DES CALCULS (tableau 1)}

1,3 et 6 :

Les paramètres propres à l'exploitation sont précisés dans le tableau en majuscules aux lignes

- 1 POIDS INDIVIDUEL DES POISSONS A LA FIN DE LA PHASE D’ÉLEVAGE,

- 3 TAUX DE SURVIE AU COURS DE LA PHASE D'ÉLEVAGE,

- 6 BIOMASSE tOTALE RÉCOLTÉ A LA FIN DE LA PHASE D'ÉleVAGE.

Les phases intermédiaires apparaissent en minuscules et les résultats recherchés sont encadrés: lignes $8,8^{\prime}$ et $8^{\prime \prime}$,

- 8 taille relative des étangs de chaque catégorie,

- 8' pourcentage de la superficie totale,

- 8" surface réelle des étangs de chaque catégorie pour une superficie totale de A ha.

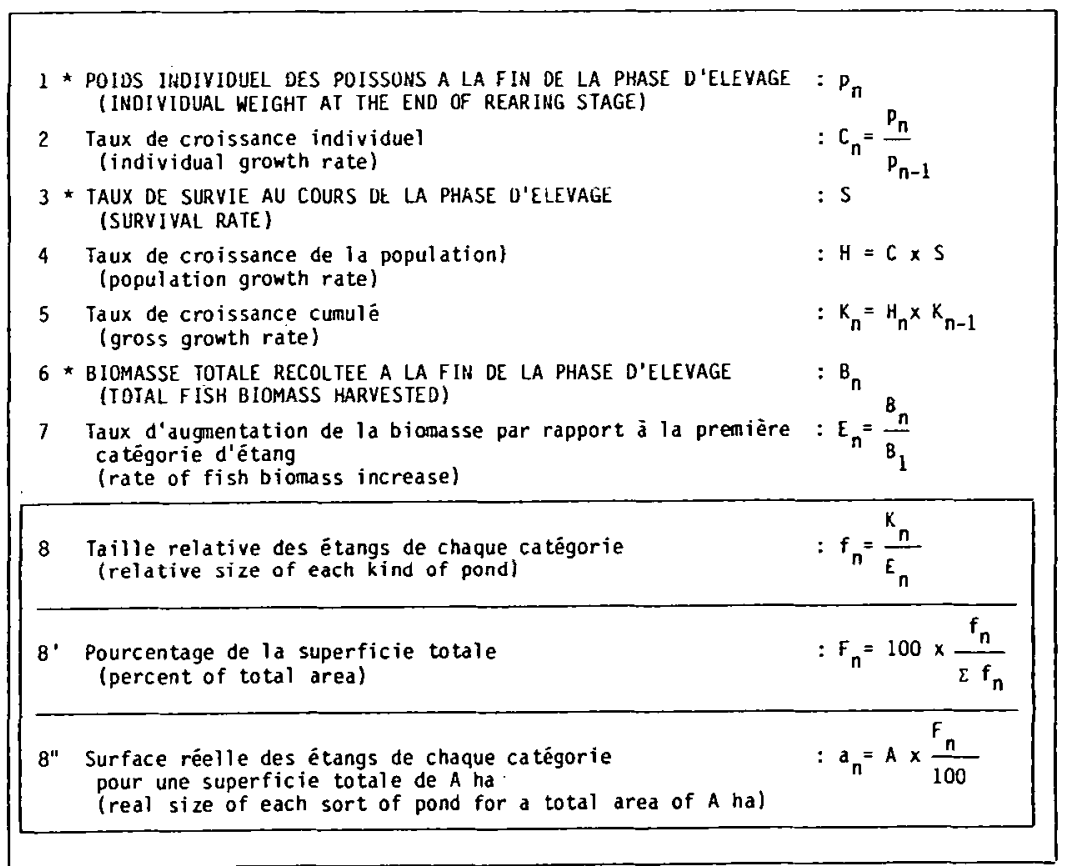

Tableau 1 : Déroulement du calcul de la répartition des surfaces.

Table 1 : Computation of surface allocation.

Une fois le calcul de la répartition des surfaces effectué, il est facile de vérifier le résultat et d'estimer les quantités de poissons de chaque classe d'âge (tableau 2). Le nombre et la biomasse des poissons récoltés à la fin d'une phase d'élevage doivent être peu différents du nombre et de la biomasse nécessaires pour l'empoissonnement de la phase suivante. Les différences qui peuvent apparaître sont dues à l'utilisation de valeurs arrondies dans le calcul.

9 Biomasse récol tẻe dans chaque catégorie (harvested Diomass)

9. Nombre de poissons récoltës dans chaque catégorie (number of fish harvested)

10 Nombre de poissons mis en charge (number of fish stocked)

$10^{\prime}$ Biomasse mise en charge dans chaque catégorie (stocked biomass)

$$
\begin{aligned}
& : 0_{n}=a_{n} \times B_{n} \\
& : L_{n}=\frac{O_{n}}{p_{n}} \\
& : M_{n}=\frac{L_{n}}{S_{n}} \\
& : N_{n}=M_{n} \times P_{n-1}
\end{aligned}
$$

Tableau 2 : Calcul des flux de poissons.

Table 2 : Fish flow computation. 


\section{4 - EXEMPLE D'UTILISATION.}

Quatre exemples reprenant différentes stratégies d'exploitation sont présentés sur le tableau 3 et la figure 1.

La connaissance de ces chiffres pour la répartition des surfaces d'une pisciculture en fonction de son projet d'exploitation en liaison avec son programme d'aménagement permet d'examiner à l'avance plusieurs points critiques, tels que :

- le bilan de son alimentation en eau.

- le choix d'un emplacement fonctionnel des différents étangs, compte tenu des caractéristiques du terrain disponible,

- les analyses prévisionnelles de l'efficacité économique du cycle de production, etc.

Tous ces calculs permettent au pisciculteur d'analyser avec une certaine précision les choix techniques et technologiques liés à son projet.

\begin{tabular}{|c|c|c|c|c|c|}
\hline \multirow{4}{*}{$\begin{array}{l}\text { Paramètres } \\
\text { (parameters) }\end{array}$} & \multirow{4}{*}{$\begin{array}{l}\text { Catégories } \\
\text { d'étangs } \\
\text { (Pond type) }\end{array}$} & \multicolumn{4}{|c|}{$\begin{array}{l}\text { Cycle de production } \\
\text { (production scheme) }\end{array}$} \\
\hline & & \multicolumn{2}{|c|}{3 ans } & \multicolumn{2}{|c|}{2 ans } \\
\hline & & \multicolumn{2}{|c|}{$\begin{array}{l}\text { Intensi té de } \\
\text { production } \\
\text { (degree of } \\
\text { intensification) }\end{array}$} & \multicolumn{2}{|c|}{$\begin{array}{c}\text { Intensi té de } \\
\text { production } \\
\text { (degree of } \\
\text { intensification) }\end{array}$} \\
\hline & & $\left\{\begin{array}{c}\text { moyenne } \\
n^{\circ} 1 \\
\text { (medium) }\end{array}\right.$ & $\begin{array}{c}\text { basse } \\
n^{\circ} 2 \\
(10 w)\end{array}$ & $\begin{array}{c}\text { moyenne } \\
n^{\circ} 3 \\
\text { (medium) }\end{array}$ & $\begin{array}{l}\text { basse } \\
n^{\circ} 4 \\
(10 w)\end{array}$ \\
\hline $\begin{array}{l}\text { Poids individuel } \\
\text { des poissons à } \\
\text { la fin de la } \\
\text { phase d'élevage } \\
\quad / \mathrm{kg} / \\
\text { (ind. weight) }\end{array}$ & $\begin{array}{l}\text { ler alevinage } \\
\text { 2ème alevinage } \\
\text { ler hivernage } \\
\text { grossissement } \\
\text { 2ème hivernage } \\
\text { engraissement }\end{array}$ & $\begin{array}{l}0,003 \\
0,04 \\
0,04 \\
0,25 \\
0,25 \\
1,25\end{array}$ & $\begin{array}{l}0,003 \\
0,04 \\
0,04 \\
0,25 \\
0,25 \\
1,25\end{array}$ & $\begin{array}{l}0,003 \\
0,08 \\
0,08 \\
- \\
- \\
1,25\end{array}$ & $\begin{array}{l}0,003 \\
0,08 \\
0,08 \\
- \\
- \\
1,25\end{array}$ \\
\hline $\begin{array}{l}\text { Coefficient de } \\
\qquad / \% / \\
\text { survie } \\
\text { (survival rate) }\end{array}$ & $\begin{array}{l}\text { ler al evinage } \\
\text { 2ème al evinage } \\
\text { ler hivernage } \\
\text { grossissement } \\
\text { 2ème hivernage } \\
\text { engraissement }\end{array}$ & $\begin{array}{l}0,5 \\
0,5 \\
0,85 \\
0,85 \\
0,9 \\
0,9\end{array}$ & $\begin{array}{l}0,5 \\
0,5 \\
0,85 \\
0,85 \\
0,9 \\
0,9\end{array}$ & $\begin{array}{l}0,5 \\
0,5 \\
0,85 \\
- \\
- \\
0,9\end{array}$ & $\begin{array}{l}0,5 \\
0,5 \\
0,85 \\
- \\
- \\
0,9\end{array}$ \\
\hline 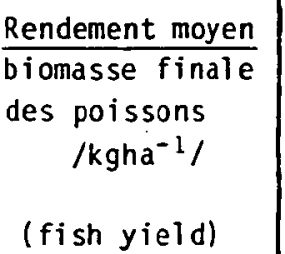 & $\begin{array}{l}\text { ler alevinage } \\
\text { 2ème al evinage } \\
\text { ler hivernage } \\
\text { grossissement } \\
\text { 2ème hivernage } \\
\text { engraissement }\end{array}$ & $\begin{array}{ll} & 150 \\
& 600 \\
3 & 000 \\
& 800 \\
3 & 000 \\
1 & 000\end{array}$ & $\begin{array}{r}150 \\
300 \\
3000 \\
350 \\
3000 \\
350\end{array}$ & $\begin{array}{l}150 \\
600 \\
3000 \\
- \\
- \\
1000\end{array}$ & $\begin{array}{l}150 \\
300 \\
3000 \\
- \\
- \\
350\end{array}$ \\
\hline
\end{tabular}

Tableau 3 : Paramètres techniques d'élevage appliqués au calcul.

Table 3 : Technical parameters used in surface computation. 

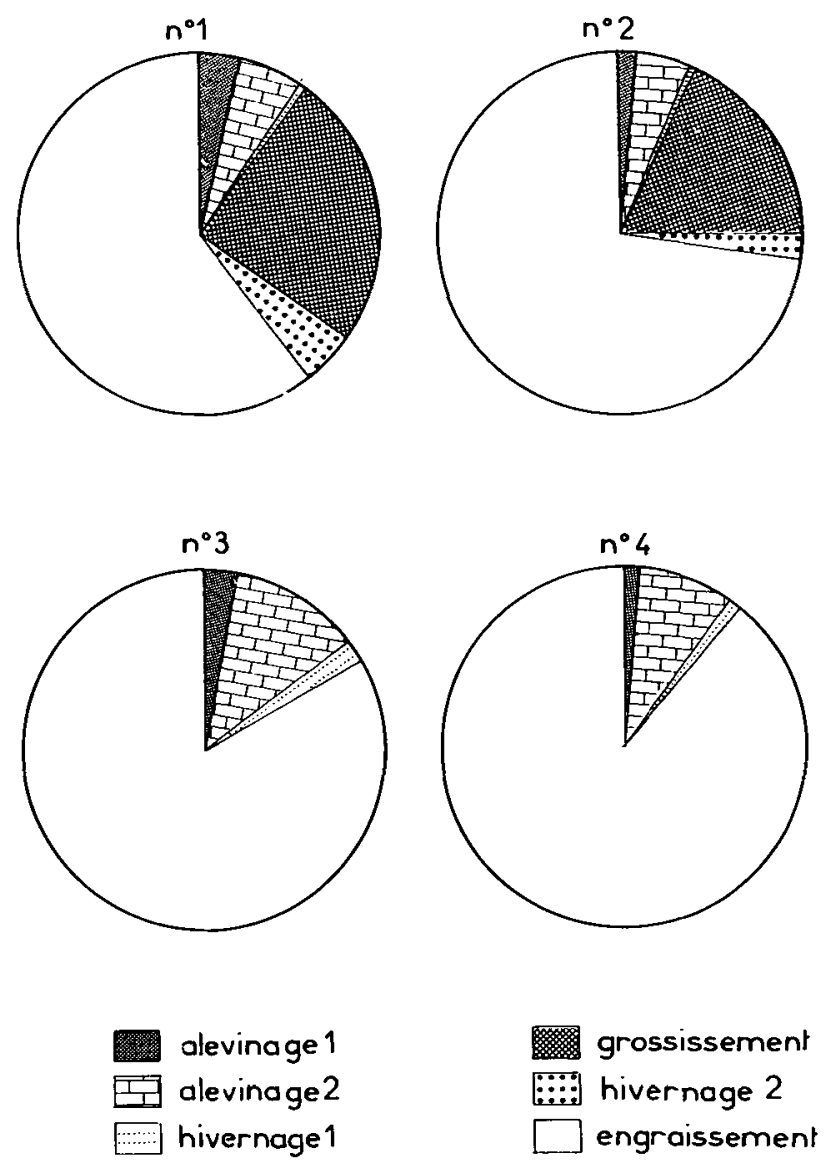

Figure 1 : Répartition des surfaces de chaque catégorie d'étang selon les différents modèles techniques exposés au tableau 3.

Figure 1 : Surface allocation of each sort of ponds with the different technical parameters shown in table 3.

\section{BIBLIOGRAPHIE}

SCHÄPERCLAUS W., 1933. Lehrbuch der Teichwirtschaft. Paul Parey. Berlin, 289 p.

SPICZAKOW T., 1932. Racjonalny stosunek powierzchni stawowych przy roznych systemach gospodarowania. Przeglad Rybacki 5 (8). Zwiazek Organizacji Rybackich. Warszawa.

WALTER E., 1934. Grundlagen der allgemeinen fischereilichen Produktionslehre. In Handbuch der Binnenfischerei Mitteleuropas 4 (5) pp. 484-544.

WIENIAWSKI J., 1963. Organizacja produkcji w gospodarstwie stawowym. Panstwowe Wydawnictwo Rolnicze i Lesne. Warszawa. pp. 511-536.

WIENIAWSKI J., 1980. Conception et construction des étangs. In Billard R., La Pisciculture en Étang, INRA Publ., Paris, 383-395. 


\section{ANNEXES}

\section{Exemples de calculs}

Ce travail permet de proposer un modèle de gestion technique simple à mettre en cuvre et d'optimiser la répartition des surfaces destinées aux différentes phases d'élevage dans le cas précis d'une pisciculture au sein de laquelle la filière de production d'une espèce est totalement maîtrisée. Ce type de calcul permet les mêmes évaluations que celles obtenues avec des formules plus classiquement retenues pour l'évaluation des empoissonnements: $N=\frac{(P-E) \times 100}{\left(p_{2}-p_{1}\right) \times S}$, où $(P-E)$ est la production nette, soit la différence de biomasse entre la pêche et l'empoissonnement, $\left(p_{2}-p_{1}\right)$ la croissance individuelle des poissons, soit la différence entre le poids moyen au début et à la fin de l'élevage et $\mathrm{S}$ le taux de survie.

Cette méthode permet de travailler plus simplement en ne tenant compte que de la biomasse totale pêchée, du poids final des poissons et de leur taux de survie et, surtout, en pouvant replacer ces calculs dans une exploitation intégrée. Elle permet, de plus, de simuler les effets d'une technique d'élevage différente, tant sur les quantités de poissons produites que sur le plan de la rentabilité économique.

En développant le calcul avec des paramètres propres à une exploitation particulière (survie, croissance et production totale à l'ha), il permet d'évaluer avec une certaine précision l'ensemble des productions et les contraintes de surfaces qui lui sont liées (exemple 1).

En France, plus particulièrement, rares sont les exploitations qui assurent cette maîtrise totale de toutes les phases d'élevage. Ce modèle de calcul peut, cependant, être adapté aux techniques employées sur l'exploitation: absence d'étang d'hivernage, absence de phases d'alevinage, cycle débutant avant l'achat de $\mathrm{C}_{0}$ ou $\mathrm{C}_{1}$, utilisation de bassins-frayère, pratique de l'étang de pose, ..., (exemple 2); I'élevage en classes d'âge mélangées ou la polyculture peuvent, de même, être pris en compte (exemple 3 ). Tout ceci, bien sûr, sous réserve que le pisciculteur connaisse bien les 3 paramètres d'élevage: production, survie et croissance pour chaque espèce et chaque classe d'âge.

II est aussi possible de prendre ce calcul à l'envers : à partir d'un fichier regroupant l'ensemble des étangs d'une pisciculture avec leurs caractéristiques de surface selon les phases d'élevage, il est possible d'optimiser l'affectation de ces étangs à une phase d'élevage particulière. Tout cela en étant en mesure de chiffrer à l'avance l'excédent ou le déficit d'une classe d'âge à la fin de la saison d'élevage, et ainsi de prendre des dispositions pour programmer des ventes ou des achats avant les empoissonnements de la saison suivante (exemples 4 et 5 ).

Tous ces éléments calculables au sein d'une exploitation peuvent bien sûr être calculés chez un ensemble de producteurs décidés à mettre en commun leurs étangs pour assurer la maitrise d'une filière sans avoir recours à des achats ou des ventes répétées à chaque saison de production.

\section{EXEMPLES}

\section{Exemple 1 : Stratégies techniques différentes}

Considérons le cas d'une pisciculture d'étang en France, où l'élevage de la carpe se déroule en 3 ans, avec une succession étang d'alevinage $\rightarrow$ étang de grossissement ( $2^{\mathrm{e}}$ été) $\rightarrow$ étang d'engraissement ( $3^{e}$ été) sans phase d'hivernage en étang spécialisé (l'empoissonnement a lieu juste après la pêche). Les étangs d'alevinage sont mis en charge avec des alevins de 5 semaines achetés à l'extérieur.

Les paramètres d'élevage dans cet exemple seront:

\begin{tabular}{|l|c|c|c|}
\hline & ler été & 2ème été & 3ème été \\
\hline poids individuel & & & \\
à la pêche & 0,02 & 0,2 & 1,2 \\
taux de survie & 30 & 60 & 80 \\
rendement & 100 & 150 & 200 \\
\hline
\end{tabular}

Le calcul permet de fixer les surfaces nécessaires pour chaque phase d'élevage : (Cf. tableau 1)

$$
\begin{array}{ll}
\text { - alevinage } & : \quad 5,1 \% \\
\text { - grossissement }: & 20,6 \% \\
\text { - engraissement }: & 74,2 \%
\end{array}
$$


Les quantités de poissons mis en jeu pendant le cycle d'élevage sur une exploitation de ce type disposant d'une surface totale de 100 ha sont alors (voir tableau 2):

\begin{tabular}{|l|c|c|c|c|}
\hline \multirow{1}{*}{} & \multicolumn{2}{|c|}{ Empoissornement } & \multicolumn{2}{c|}{ Récol te } \\
\cline { 2 - 5 } & $\begin{array}{c}\text { Nombre } \\
\mathrm{u}\end{array}$ & $\begin{array}{c}\text { Biomasse } \\
\mathrm{kg}\end{array}$ & $\begin{array}{c}\text { Nombre } \\
\mathrm{u}\end{array}$ & $\begin{array}{c}\text { Biomasse } \\
\mathrm{kg}\end{array}$ \\
\cline { 2 - 6 } & $85000 \mathrm{C}_{0}$ & - & $25700 \mathrm{C}_{1}$ & 510 \\
Al evinage & $25700 \mathrm{C}_{1}$ & 510 & $15450 \mathrm{C}_{2}$ & 3100 \\
Grossissement & $15450 \mathrm{C}_{2}$ & 3100 & $12300 \mathrm{C}_{3}$ & 14800 \\
\hline Engraissement & 15 & & & \\
\hline
\end{tabular}

Ces chiffres arrondis permettent d'évaluer un ensemble de contraintes liées à la bonne réalisation du programme de production:

- I'achat de 85.000 alevins de 5 semaines,

- la prévision des pêches où un volume connu de poisson sera en transit $\left(\simeq 26.000 \mathrm{C}\right.$, et $\left.16.000 \mathrm{C}_{2}\right)$, ce qui permet de prévoir le matériel nécessaire pour le stockage et le transport.

- la production finale commercialisable $\left(\simeq 12.300 \mathrm{C}_{3}\right.$ pour une quinzaine de tonnes). ce qui permet d'effectuer un calcul de rentabilité estimatif.

\section{Exemple 2 : Utilisation de bassins-frayère}

Considérons le type d'élevage décrit dans l'exemple 1, dans lequel la production des larves, servant à la mise en charge des étangs de premier été, est assurée par des bassins-frayère.

Les caractéristiques de ces bassins-frayère où l'on place une "pose" de géniteurs dans $50 \mathrm{~m}^{2}$ sont les suivantes : considérant qu'une femelle produit en moyenne 60.000 œufs par $\mathrm{kg}$ dont $70 \%$ sont fécondés et $30 \%$ donnent des larves et que les géniteurs pèsent $4 \mathrm{~kg}$ :

$$
\begin{array}{lll}
\text { - survie } & : 20 \% \\
\text { - poids final }: & 0,000.003 \mathrm{~kg} \text { (330 larves par } \mathrm{ml} \text { ) } \\
\text { - production : } & 30 \mathrm{~kg} \mathrm{ha}^{-1}
\end{array}
$$

Le calcul repris avec cette série de bassins supplémentaires donne des résultats très peu différents de ceux de l'exemple 1 : les bassins-frayère ne représentent que $0.009 \%$ de la superficie totale, soit $90 \mathrm{~m}^{2}$ sur 100 ha. Bien que ne faisant varier que dans une infime proportion les surfaces et les quantités de poissons en élevage, l'introduction de ce type de bassins dans le calcul permet d'estimer le nombre de géniteurs nécessaires pour approvisionner l'exploitation: dans ce cas 2 femelles sur 4 mâles semblent suffisants ; il est cependant nécessaire d'en prévoir le double pour tenir compte d'un pourcentage d'ovulation des femelles de $50 \%$.

\section{Exemple 3: Pratique de la polyculture}

Considérons maintenant une exploitation où est pratiquée une polyculture de carpes et tanches

\begin{tabular}{|c|c|c|c|c|c|c|}
\hline & \multicolumn{3}{|c|}{ carpes } & \multicolumn{3}{|c|}{ tanches } \\
\hline & ler été & Zème été & 3ème été & ler été & 2ème été & Jème été \\
\hline $\begin{array}{ll}P_{n} & (k g) \\
S_{n} & (q) \\
B_{n}^{n} & \left(k g \quad h a^{-1}\right)\end{array}$ & $\begin{array}{c}0,02 \\
30 \\
100\end{array}$ & $\begin{array}{r}0,2 \\
60 \\
150\end{array}$ & $\begin{array}{r}1,2 \\
80 \\
200\end{array}$ & $\begin{array}{c}0,005 \\
20 \\
30\end{array}$ & $\begin{array}{c}0,05 \\
60 \\
50\end{array}$ & $\begin{array}{l}0,3 \\
80 \\
80\end{array}$ \\
\hline
\end{tabular}
de même classe d'âge.

Les caractéristiques de chaque production seraient par exemple: 
Pour calculer la surface nécessaire pour une production en polyculture, il faut considérer que chaque espèce occupe des parties d'étangs différentes qu'il faut ensuite additionner. Le calcul n'est cependant valable que si l'on tient compte des effets réciproques des espèces les unes sur les autres dans l'énoncé des paramètres retenus : ainsi, dans les cas de polyculture avec des carpes chinoises, on peut considérer que les rendements en carpes communes sont augmentés de 20-30\% pour tenir compte de l'effet positif des carpes chinoises sur le milieu et donc sur la productivité de l'étang.

Inversement, dans le cas d'une polyculture de poissons de petite taille avec des carnassiers, il est nécessaire pour faire varier le taux de survie par rapport à une monoculture pour tenir compte de la prédation.

Dans le cas de la polyculture carpe + tanche prise en exemple, les résultats en surfaces nécessaires sont les suivants:

$5.8 \%$ pour le $1^{\text {er }}$ été $\left(C_{0}+T_{0} \rightarrow C_{1}+T_{1}\right)$

$22,2 \%$ pour le $2^{\theta}$ été $\left(C_{1}+T_{1} \rightarrow C_{2}+T_{2}\right)$

$72,0 \%$ pour le $3^{e}$ été $\left(C_{2}+T_{2} \rightarrow C_{3}+T_{3}\right)$

Les quantités de poissons mises en jeu sur une exploitation de ce type disposant d'une surface totale de 100 ha sont alors, en valeurs arrondies:

\begin{tabular}{|c|c|c|c|c|c|c|c|c|}
\hline & \multicolumn{4}{|c|}{ Empoissonnement } & \multicolumn{4}{|c|}{ Pêche } \\
\hline & \multicolumn{2}{|c|}{ Carpe } & \multicolumn{2}{|c|}{ Tanche } & \multicolumn{2}{|c|}{ Carpe } & \multicolumn{2}{|c|}{ Tanche } \\
\hline & N & B & $\mathbf{N}$ & B & N & B & N & B \\
\hline ler èté & 96600 & - & 175000 & - & 28000 & 550 & 34000 & 180 \\
\hline 2ème èté & 28000 & 550 & 38000 & 180 & 16600 & 3200 & 24000 & 1200 \\
\hline 3ème été & 16600 & 3200 & 24000 & 1200 & 12000 & 14500 & 19000 & 5800 \\
\hline
\end{tabular}

\section{Exemple 4 : Application à une pisciculture existante}

Dans le cas d'une pisciculture existante disposant d'une dizaine d'étangs sur lesquels les paramètres d'élevage applicables sont ceux décrits à l'exemple 1, le problème posé est d'assigner à chaque étang une phase d'élevage afin qu'il s'intègre dans la filière de production.

Ces clix étangs ayant des surfaces de:

$a: 20$ ha, $b: 20$ ha, $c: 10$ ha, $d: 8$ ha, $e: 5$ ha, $f: 30$ ha, $g: 60$ ha, $h: 45$ ha, $i: 15$ ha, $j: 7$ ha, soit : 220 ha de surface totale,

les superficies nécessaires à chaque phase d'élevage sont: *

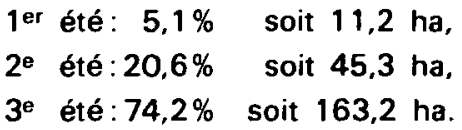



calculées :

Différentes répartitions peuvent être alors adoptées pour approcher au mieux ces valeurs

\begin{tabular}{|c|c|c|c|}
\hline répartition & ler été & Zème èté & 3ème ēté \\
\hline option 1 & $\begin{aligned} & e+j \\
= & 12 \mathrm{ha}\end{aligned}$ & $\begin{array}{c}h \\
=45 \mathrm{ha}\end{array}$ & $\begin{array}{l}a+b+c+d \\
+f+g+i \\
=163 \mathrm{ha}\end{array}$ \\
\hline option 2 & $\begin{array}{c}\mathrm{c} \\
=10 \mathrm{ha}\end{array}$ & $\begin{aligned} & f+i \\
= & 45 \mathrm{ha}\end{aligned}$ & $\begin{array}{l}a+b+d+e \\
+g+h \\
=165 \mathrm{ha}\end{array}$ \\
\hline option 3 & $\begin{aligned} & d+e \\
= & 13 \mathrm{ha}\end{aligned}$ & $\begin{array}{l}b+j+j \\
=47 \mathrm{ha}\end{array}$ & $\begin{array}{l}a+c+f \\
+g+h \\
=160 \mathrm{ha}\end{array}$ \\
\hline etc. & & & \\
\hline
\end{tabular}

\section{Exemple 5 :}

Dans ce cas, au contraire, l'exploitation ne dispose que de peu d'étangs qui ont, de plus, des surfaces similaires:

$a: 30$ ha, $b: 45$ ha, $c: 60$ ha, $d: 35$ ha, soit: 170 ha.

Avec les paramètres retenus pour l'exemple 1, les surfaces affectées à chaque phase devraient être :

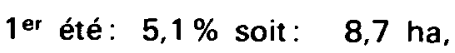

$$
\begin{aligned}
& 2^{\mathrm{e}} \text { été : } 20,6 \% \text { soit: } 35,1 \text { ha, } \\
& 3^{\text {e }} \text { été: } 74,2 \% \text { soit: } 126,2 \text { ha. }
\end{aligned}
$$

Dans ce cas, il n'apparaît pas possible d'affecter à chaque étang une phase d'élevage intégrée au processus de production. Plusieurs possibilités peuvent alors être retenues:

a) ne pas effectuer la phase $1^{\text {er }}$ été et acheter les $C_{1}$ nécessaires à l'empoissonnement de l'étang " $d$ " $\left(\simeq 45.000 \mathrm{C}\right.$, pour $900 \mathrm{~kg}$ ), et utiliser les étangs " $a$ ", " $b$ " et "c", soit 135 ha pour le $3^{e}$ été.

b) réaliser l'alevinage sur l'étang " $a$ "; il reste donc 140 ha que l'on peut répartir ainsi : "b" pour le $2^{e}$ été (soit 35 ha au lieu de 29 calculés), et "c" et "d" pour le $3^{\text {e }}$ été (soit 105 ha au lieu de 104 calculés), en prévoyant une vente de $75 \%$ des $C_{1}$ récoltés dans l'étang "a" $\left(\simeq 29.000 C_{1}\right.$, pour $\left.580 \mathrm{~kg}\right)$.

c) etc. 